\title{
Routine Screening for HIV in an Urban Emergency Department During the COVID-19 Pandemic
}

\author{
Kimberly A. Stanford ${ }^{1}$ - Eleanor E. Friedman ${ }^{2}$. Jessica Schmitt ${ }^{2}$. Thomas Spiegel ${ }^{1}$. Jessica P. Ridgway ${ }^{2}$. \\ Michelle Moore $^{2} \cdot$ Michelle Taylor $^{2} \cdot$ David Pitrak $^{2} \cdot$ Moira C. McNulty $^{2}$
}

Published online: 2 May 2020

(c) Springer Science+Business Media, LLC, part of Springer Nature 2020

The emergency department (ED) has been identified as a key location for HIV screening, with many new diagnoses resulting from non-targeted ED screening programs [1]. The COVID-19 pandemic has greatly affected ED operations across the country. Many EDs have seen dramatic increases in patient volumes, while others have experienced a lull, as only the most serious cases continue to arrive at the ED. Preparedness for and management of COVID-19 are clearly emergent public health priorities, but other important public health initiatives, such as expanded HIV screening and linkage to care, must continue. Routine HIV screening in the ED faces many barriers in normal times, due to competing priorities and complexities of implementation [2]. The COVID-19 pandemic presents further challenges to routine screening programs. We describe how our urban ED in Chicago, with advance planning, was able to continue routine HIV screening in the face of the COVID-19 pandemic.

\section{HIV Screening Program and ED Design}

The University of Chicago has had a program for expanded HIV testing and linkage to care at our hospitals and clinics since 2011. Recently that program has placed special emphasis on screening in the ED, utilizing automated electronic medical record (EMR) reminders to support test ordering. All ED patients under age 65 who have no known diagnosis of HIV and have not been tested for HIV in the last year are eligible for screening. The HIV care team assumes all

Kimberly A. Stanford

kstanford@medicine.bsd.uchicago.edu

1 Section of Emergency Medicine, Department of Medicine, University of Chicago, Chicago, IL, USA

2 Section of Infectious Diseases and Global Health, Department of Medicine, University of Chicago, Chicago, IL, USA responsibility for positive test result notification, linkage to care, and initiation of antiretroviral therapy (ART).

In preparation for the COVID-19 pandemic, our ED quickly established a large temporary space for screening, testing, and treatment of patients with influenza-like illness (ILI) who were considered likely to be discharged home. With support of ED leadership, continued HIV screening of patients was incorporated into the design of this secondary ED space, with a station for lab draws designated for HIV screening. This space otherwise has extremely limited diagnostic capacity, with capabilities only for chest x-ray, EKG, viral swab, and strep swabs (Fig. 1). There was initially some resistance to HIV testing on the part of staff, many of whom felt that COVID-19 should be the sole priority. However, ED leadership offered provider education regarding the overlap of symptoms between COVID-19 and acute HIV and emphasized the opportunity for wide community screening. This messaging was positively received by ED staff. Outside of the temporary space, workflows and HIV screening continued as normal with automated EMR alerts and test orders.

We assessed routine HIV testing rates in the ED from January 1, 2020, until April 18, 2020. For this analysis, we considered March 5, 2020, to be the beginning of the pandemic period, as this was the date of the first case of COVID-19 in the City of Chicago. All dates prior to March 5, 2020, are considered pre-pandemic. We describe the outcomes of all patients newly diagnosed with HIV during the pandemic, including days to notification, days to first medical visit, and days to ART initiation.

\section{Testing Trends}

During the first month and a half of the COVID-19 pandemic, the ED conducted 1789 HIV tests, screening 20.8\% of the total $8616 \mathrm{ED}$ visits during this time. This was not significantly different from testing performed during the 
Fig. 1 Diagram of temporary ED space for ILI patients

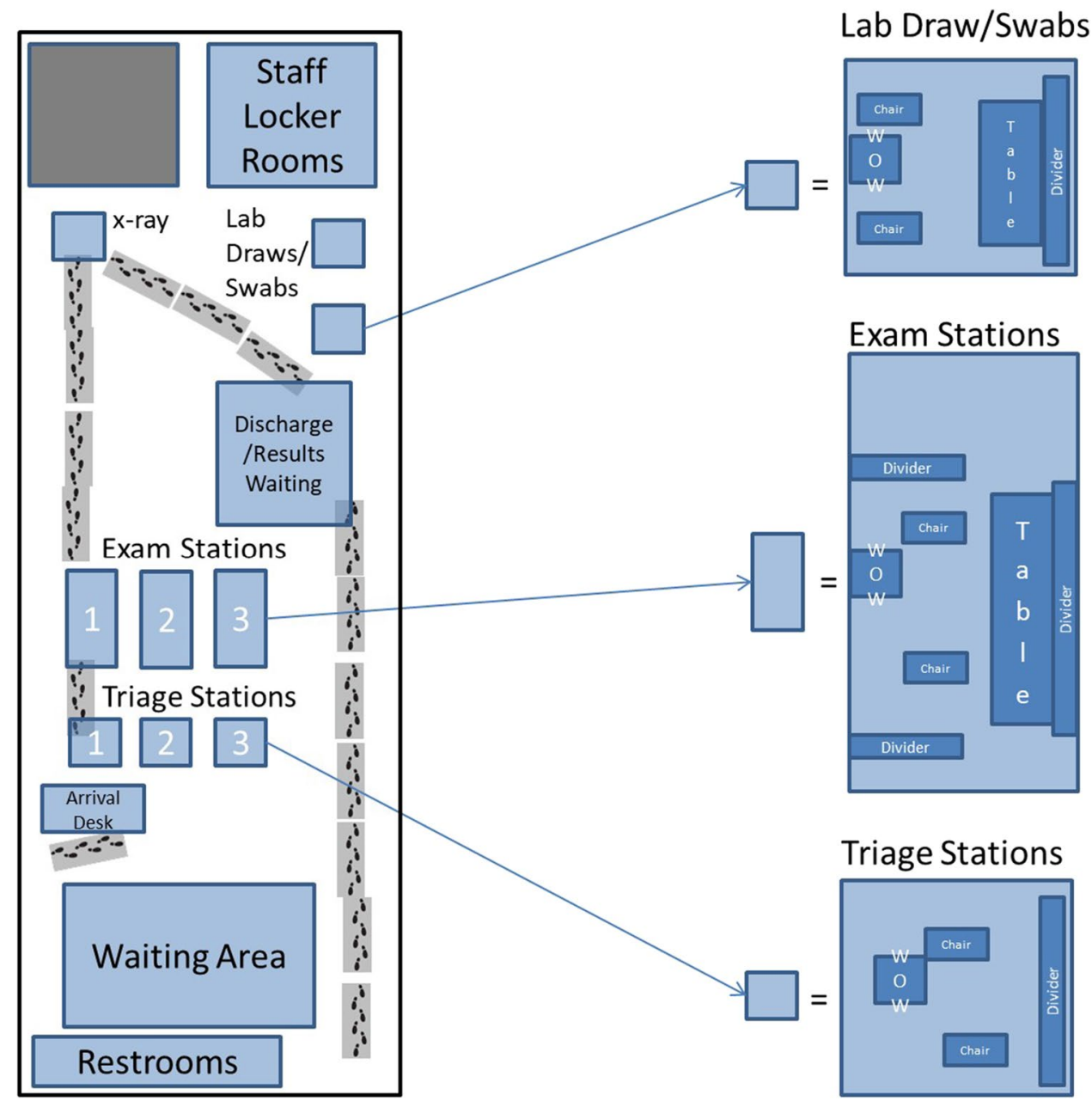

pre-pandemic period, when 3247 tests were performed, representing $22.4 \%$ of the total 14,524 ED visits. COVID19 testing at our hospital began on March 10th with $1.8 \%$ of ED visits, and rose to $53.5 \%$ of daily ED visits on April 16, 2020 (Fig. 2).

From March 5, 2020, to April 18, 2020, six patients with a new diagnosis of HIV were identified via routine HIV screening in the ED. The patients were young (median age 23 years, range 21-30), predominantly African American $(80 \%)$, and all were uninsured or had Medicaid. There were two cis-gender heterosexual women, three men who have sex with men (MSM), and one male injection drug user. The median baseline viral load was 84,650 copies $/ \mathrm{mL}$. Patients were notified of their new diagnosis a median of 1.5 days after testing (range 1-4 days), started ART after a median of 1 day (range 1-4), and $80 \%$ attended a comprehensive care clinic visit after a median of 1 day (range 1-4), with one visit being a telehealth visit. One patient was notified of their results, but has not attended an appointment at time of publication.

Two of these patients (1 woman and 1 man) had acute HIV infection (AHI) and presented with ILI symptoms. Both tested negative for COVID-19 and other respiratory viruses. An additional patient was admitted for cellulitis and diagnosed with advanced HIV disease with a CD $4<200 \mathrm{~mm}^{3}$. All three were linked to care and initiated ART within $24 \mathrm{~h}$ of diagnosis.

\section{Lessons Learned}

There has been significant progress in reducing the number of new HIV diagnoses, and the goal of eliminating new HIV transmission is closer than ever [3]. In order not to lose ground, it is important to maintain HIV screening and linkage to care, even in the face of the COVID-19 pandemic. HIV elimination initiatives in Illinois and nationwide require expansion and intensification of HIV screening and linkage to care efforts $[4,5]$. There should be no reduction in this important effort, even with the obstacles posed by COVID19. While there are many obvious challenges to EDs during the pandemic, there are also possible opportunities to reach patients who otherwise would not have sought care. Maintaining high levels of routine HIV screening in the ED is 


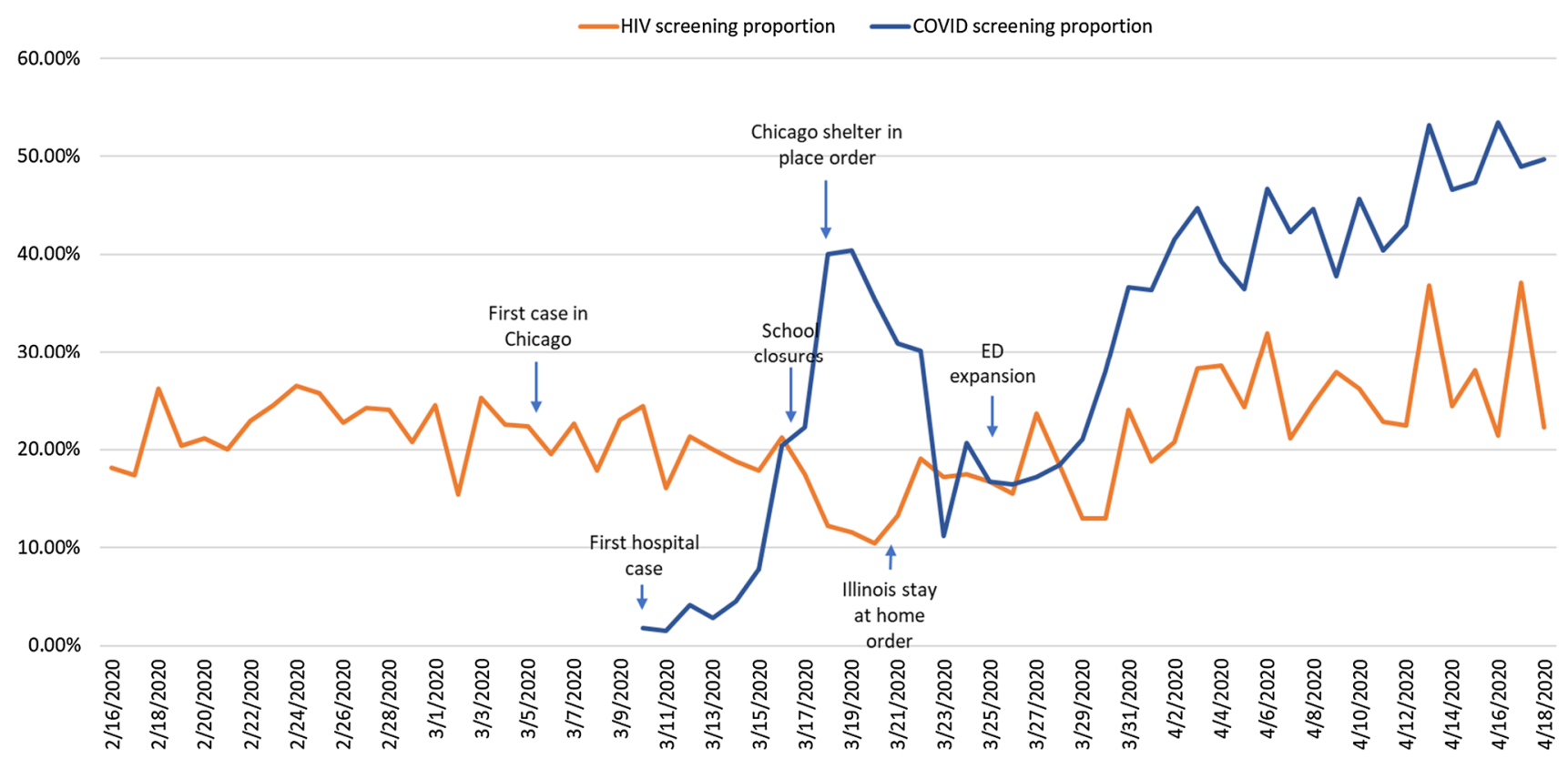

Fig. 2 HIV and COVID-19 tests by date

crucial, as other community-based organizations and health clinics may have ceased to offer in-person evaluations and HIV screening, making it even more difficult to be tested for HIV during the COVID-19 pandemic [6, 7].

We have demonstrated that the protocols for expanded HIV screening and linkage to care can continue during a pandemic. Our ED was able to continue to screen at a high level after the emergence of COVID-19 by planning to have blood draws and HIV screening as part of a fast track for patients with ILI. Provider education emphasizing the importance of continued screening and overlap of symptoms between acute HIV and COVID-19 ensured staff buy in. The two patients with AHI presented with ILI and would likely have been misdiagnosed without the inclusion of HIV testing in the new ED space. AHI is a priority for care programs due to their high viral loads and high risk of transmission to others, increasing the importance of the identification of these cases [8].

Other EDs, as well as other patient care sites that see a population at risk for HIV infection, should adopt a care model for patients with ILI that includes phlebotomy for combination HIV Antigen/Antibody testing, as well as nasopharyngeal swabs for COVID-19 and other respiratory viruses. Partnerships between front line ED providers and HIV care providers to ensure rapid initiation of comprehensive care are key to the success of these programs, especially at times of crisis.

\section{References}

1. Rothman RE, Lyons MS, Haukoos JS. Uncovering HIV infection in the emergency department: a broader perspective. Acad Emerg Med. 2007;14(7):653-7.

2. Galbraith JW, Willig JH, Rodgers JB, Donnelly JP, Westfall AO, Ross-Davis KL, Heath SL. Evolution and escalation of an emergency department routine, opt-out HIV screening and linkage-tocare program. Publ Health Rep. 2016;131(1_suppl):96-106.

3. Centers for Disease Control and Prevention. HIV Surveillance Report, 2018 (Preliminary); vol. 30. https://www.cdc.gov/hiv/ library/reports/hiv-surveillance.html. Published November 2019. Accessed 23 April 2020.

4. Getting to Zero Illinois: 2019-2023 Plan. https://gtzillinois.hiv/ wp-content/uploads/2019/06/GTZ-IL-plan-English-06062019. pdf. Published May 2019. Accessed 23 April 2020.

5. NASTAD. Ending the HIV epidemic: jurisdictional plans. https:// www.nastad.org/maps/ending-hiv-epidemic-jurisdictional-plans. Accessed 23 April 2020.

6. Jiang H, Zhou Y, Tang W. Maintaining HIV care during the COVID19 pandemic. The Lancet HIV. 2020;S2352-3018(20):30105-3.

7. Hoff T, Kates J, Dawson L, Kistler R. Kaiser Family Foundation. Managing HIV during COVID-19: working to end one epidemic while confronting another. https://www.kff.org/coronavirus-polic y-watch/managing-hiv-during-covid-19-working-to-end-oneepidemic-while-confronting-another/. Accessed 23 April 2020.

8. White DA, Giordano TP, Pasalar S, Jacobson KR, Glick NR, Beverly ES, Mammen PE, Hunt BR, Todorovic T, Moreno-Walton L, Adomolga V. Acute HIV discovered during routine HIV screening with HIV antigen-antibody combination tests in 9 US emergency departments. Ann Emerg Med. 2018;72(1):29-40.

Publisher's Note Springer Nature remains neutral with regard to jurisdictional claims in published maps and institutional affiliations. 